\title{
Efficiency of Scattering Algorithm with Overset Grid Generation for Detection of Buried Object
}

\author{
Deanne Anak Edwin ${ }^{1}$, Shafrida Sahrani ${ }^{* 2}$, Kismet Hong Ping ${ }^{3}$ \\ ${ }^{1,2,3}$ Department of Electrical and Electronic,Faculty of Engineering, Universiti Malaysia Sarawak \\ 94300, Kota Samarahan, Sarawak, Malaysia \\ *Corresponding author, email: sshafrida@unimas.my
}

\begin{abstract}
The safety of building and civil structures affected by seismic waves emanating from the earthquakes has always been of concern to the general public. Post-hazard assessments must be conducted to ensure the integrity of the affected buildings, infrastructures and lands before continuing usage. Extensive research on damage detection of buildings and civil structures has recently been performed. However, it remains a significant problem and yields an unpredictable outcome as in most cases the results from a certain method applied to one structure are not valid for other structures. The ForwardBackward Time-Stepping (FBTS) technique utilizing FiniteDifference Time-Domain (FDTD) method and Overset Grid Generation (OGG) method is proposed to solve the problem. This paper investigates the accuracy of the proposed method by the analysis with various ratio of grid size between the main mesh and sub mesh. The signal analysis is carried out for inverse scattering with empty grid that overlapped on the main mesh. It is shown that the smaller grids give more accuracy for the results.
\end{abstract}

Keywords-Overset Grid Generation; inverse scattering; buried object;Finite Difference Time Domain; Forward Backward Time Stepping; Structural Health Monitoring

\section{INTRODUCTION}

Microwave inverse scattering technique is used to determine location, shape and the material properties of unknown objects that are scattered by the objects. However, this technique has ill-posed problem due to the non-linearity and limited measurement parameters available enforced by the problem geometry[1], [2]. Forward Backward Time Stepping (FBTS) utilizing Finite Difference Time Domain (FDTD) with Overset Grid Generation (OGG) method has been proposed previously to solve the inverse scattering problem. This method has also been proposed to improve the image reconstruction for the detection of unknown object [3]. However, the investigation is still at the initial stage.

In this paper, the proposed method is extended to investigate its efficiency by analyzing the various ratio of the grid size between the main mesh and sub-mesh. Note that in this analysis, the main mesh is set as grid domain and submesh as the buried object and in stationary case. The analysis is investigated in free space to determine the accuracy of the grid before it is modeled as an object. The measured signals for each ratio of grid size, $R$ are compared for the numerical errors analysis.
Extensive research on damage detection of buildings and civil structures has recently been performed [4]-[6]. The damage of the building and civil structures can be detected at a very early stage with microwave inverse scattering technique [1]. In this paper, the proposed method is applied into Structural Health Monitoring (SHM) for future work. SHM has been widely used for monitoring and evaluating structural components especially in civil structures[6]-[8]. The health monitoring should be done for the infrastructures that have been affected by the nature disaster such as the earthquake that happened in Malaysia recently.

\section{FORWARD BACKWARD TIME STEPPING WITH OVERSET GRID} GENERATION METHOD

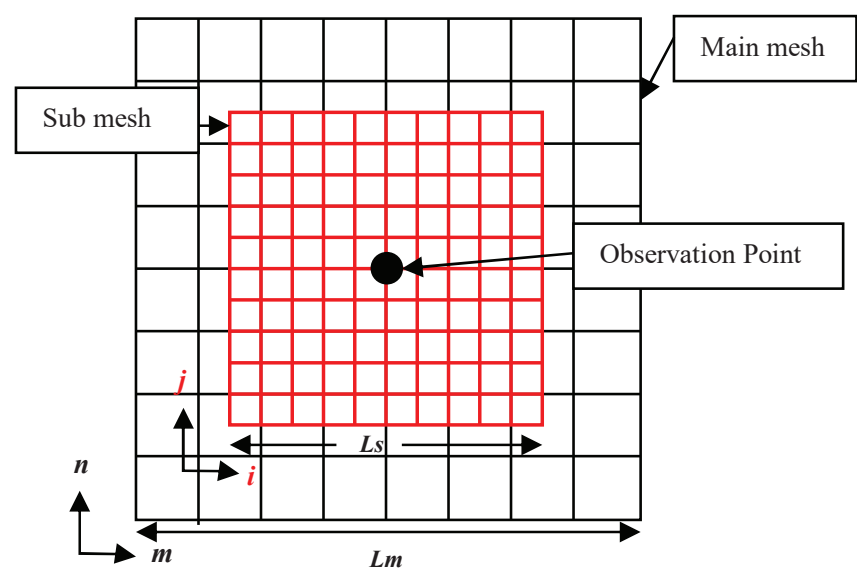

Fig. 1 Numerical model of the Overset Grid Generation method

Overset Grid Generation method has been used mainly in computational fluid dynamics (CFD) [9],[10]. Fig. 1 illustrates the numerical model of the Overset Grid Generation (OGG) method. This method basically consists of main mesh and a sub mesh that overlapped one another. Each of the grid components in the sub mesh can be calculated separately from the main mesh [10]. This method used bilinear interpolation in order to update and exchange the information of the overlapped data of sub mesh and main mesh. 